\title{
Is there a Linkage between Celiac Disease and Adverse Pregnancy Outcomes?
}

\author{
Nicoletta Di Simone $e^{1,2 *}$, Matteo Gratta² and Giovanni Scambia ${ }^{1,2}$ \\ ${ }^{1}$ Dipartimento Scienze della Salute della Donna e del Bambino, UOC di Ostetricia e Patologia Ostetrica, Fondazione \\ Policlinico Universitario A. Gemelli IRCCS, Rome, Italy \\ ${ }^{2}$ Istituto di Ginecologia e Ostetricia, Università Cattolica del Sacro Cuore, Rome, Italy
}

\begin{abstract}
Celiac disease is an autoimmune disorder that occurs in genetically predisposed people in which the ingestion of gluten leads to a damage in the small intestine that clinically presents with malabsorption related symptoms. However it is known that celiac disease can also be the underlying cause of several non-gastrointestinal symptoms. This review summarizes the studies and data acquired on the relationship between celiac disease and gynecological/obstetric disorders. Over the past few decades numerous studies highlighted the existence of a linkage between celiac disease and gynecological/obstetric disorders such as delayed menarche, early menopause, amenorrhea or infertility and adverse pregnancy outcomes. Although much has been reported on such a linkage, much remains to be understood about the pathogenic mechanisms underlying such clinical presentations in patients with celiac disease. Studies conducted on the subject showed intestinal malabsorption, direct role of gliadin, coagulation alterations, immune-modulated mechanisms and endometrial inflammation as main triggers of gynecological/obstetric disorders in celiac patients. At the present, however, the knowledge of such mechanisms is insufficient and further studies are needed to gain a more thorough understanding of the matter.
\end{abstract}

\section{Keywords}

Celiac disease, Infertility, Pregnancy, Autoimmunity, Personalized medicine

\section{Introduction}

Celiac disease (CD) is an autoimmune enteropathy which affects as many as $1 \%$ of the population worldwide [1]. The disease is triggered by and abnormal immune response to dietary gluten, a protein component found in wheat, barley and rye, and it only occurs in genetically susceptible individuals. The genetic susceptibility to develop $C D$ is known to be carried by HLA class II molecules DQ2 or DQ8, responsible for presenting disease-related peptides to T lymphocytes.

Of the $1 \%$ of the general population affected by the disease only a percentage of $20-50 \%$ shows subjective symptoms [2]. Although the classical form of CD presents with malabsorption related symptoms such as chronic diarrhea, steatorrhea, abdominal distension, nausea, vomiting, anemia and fatigue, $C D$ can also be the underlying cause of several non-gastrointestinal symptoms which can lead to a delay in the diagnosis of the disease itself [3]. Among such nongastrointestinal symptoms of particular interest are disorders of fertility, such as delayed menarche, early menopause, amenorrhea or infertility, and adverse pregnancy outcomes, such as recurrent abortions, intrauterine growth restriction (IUGR), small for gestational age (SGA) babies, low birthweight (LBW) babies or preterm deliveries $[3,4]$.
The aim of this review is to highlight what has been understood in the last decade about the existing linkage between $C D$ and gynecological/obstetrical disorders, with particularly focusing on pregnancy complications and outcomes, and on the underlying molecular pathogenic mechanisms.

\section{Disorders of Fertility}

The first description of the existence of an association between CD and disorders of fertility was made by Morris, et al. in 1970 when they first described three infertile patients with $\mathrm{CD}$ who became pregnant after having been on a gluten free diet [5]. Since then, further investigation has been conducted on such a linkage.

*Corresponding author: Nicoletta Di Simone, Dipartimento Scienze della Salute della Donna e del Bambino, UOC di Ostetricia e Patologia Ostetrica, Fondazione Policlinico Universitario A. Gemelli IRCCS, Largo F. Vito 1, 00168 Roma, Italy

Accepted: November 19, 2018

Published online: November 21, 2018

Citation: Simone ND, Gratta M, Scambia G (2018) Is there a Linkage between Celiac Disease and Adverse Pregnancy Outcomes?. J Gastroenterol Res 2(1):89-92 
CD has been found in $4-8 \%$ of patients with unexplained infertility [5] and it has been shown that CD has an increased prevalence particularly during the fertile period [6]. These patients often present with no gastrointestinal symptoms, a fact that leads to a delay in the diagnosis of $C D$ and in its treatment. Interestingly, it has been observed that fertility was reduced in the two years preceding the diagnosis and that it returned to normal ranges following diagnosis and treatment of CD [4]. However, a normalization of the fertility rate wasn't seen in all women, since the potential of a gluten free diet to have a positive effect on fertility is related to the grade of improvement of the nutritional imbalance, seen in $C D$, that the diet provides [6].

Moreover, several studies have reported an increased incidence of menstrual cycle disorders in women with $C D$ versus healthy controls [4] including delayed menarche, early menopause, amenorrhea, oligomenorrhoea, dysmenorrhoea and menorrhagia. In a case-control study conducted by Collins, et al. [7] the age of menarche in celiac girls has been shown to be delayed for more than 2 years and in another study Sher and Mayberry found that the mean age at menopause in CD patients was 47.6 compared to 50.1 in controls [8]. As far as amenorrhea is concerned, in a Ferguson, et al. study [9] amenorrhea has been shown to be more common in the celiac group.

\section{Adverse Pregnancy Outcome}

Maternal $C D$ is known to be associated with adverse pregnancy outcomes such as recurrent miscarriage, low birthweight, small for gestational age (SGA), intrauterine growth restriction (IUGR) and pre-term birth [4]. Several studies have been conducted on the subject from which interesting findings have emerged.

A study by Gasbarrini, et al. [10] showed that women with undiagnosed $C D$ present with an higher risk of recurrent miscarriage that rises up to nine times compared with treated patients. In a study by Ciacci, et al. [11], it was shown that putting the patients on a gluten free diet leads to a reduction of the relative risk of miscarriage by approximately nine times, however it must be taken into consideration that such case-control study was limited by small numbers.

Among adverse pregnancy outcomes, IUGR is one of the major concerns as it is responsible for a 5 - 20-fold increase in perinatal mortality and it has been implicated in a higher risk of perinatal morbidity and life-long consequences [4]. Studies on the association between maternal CD and IUGR have reported a considerable variation in the odds ratios, ranging between 1.6 and 6.0 [11-13]. Such studies also found variations in treated versus untreated $C D$ [4].

Numerous studies have analyzed the relation between CD and adverse pregnancy outcomes such as pre-term delivery and low weight children at birth. These studies showed that CD women have a higher prevalence of pre-term delivery [14], and in case of an undiagnosed CD, women also have a higher risk of low birth weight (<2500 g) [13] and of SGA infants [15].

\section{Underlying Pathogenic Mechanisms}

In female patients with $C D$, the disease may lead to disorders of fertility and pregnancy alterations through a variety of different, still not properly and completely understood, mechanisms.

\section{Intestinal malabsorption}

Undiagnosed CD, as a permanent autoimmune enteropathy, leads to a small intestine mucosal inflammation and damage resulting in a condition of intestinal malabsorption that may cause folic acid, vitamin $B_{12}$, fat-soluble vitamins, iron and zinc deficiencies [4]. Among these, zinc deficiency may alter the synthesis and secretion of luteinizing hormone (LH) and folliclestimulating hormone (FSH) leading to an alteration of the hypothalamic-hypophyseal-ovarian axis, secondary amenorrhea and spontaneous abortion $[4,16]$. Folic acid deficiency, owing to the action of such vitamin in nucleic acid metabolism, leads instead to a negative impact on rapidly proliferating tissue in the embryo $[2,4]$. Among women with $\mathrm{CD}$, however, malabsorption and its effects on fertility and pregnancy has been found to be variable [4].

\section{Direct role of gliadin}

Two studies have directly investigated the role of gliadin, the alcohol soluble fraction of gluten and the primary antigen leading to an inflammatory reaction in the small intestine in CD. These studies showed that gliadin is responsible for the induction of an inflammatory reaction characterized by the production of cytokines [13] that may have negative effects on the fetus [17]. High levels of inflammatory cytokines IL-6 and CRP in amniotic fluid, but not plasma, and elevated IL-6 levels in cervicovaginal fluid were shown to be strongly associated with an increased risk of spontaneous preterm birth [17].

Furthermore, gliadin itself can activate peripheral blood $T$ cells, as measured by proliferation, expression of the activation markers and cytokine secretion (interferon- $\gamma$ and interleukin-2), which may affect the intrauterine environment [18]. In fact, aberrant expression of pro-inflammatory, antiinflammatory, and angiogenic cytokines during implantation window is one of the key factors that adversely affect endometrial development, as evidenced by the inadequate expression of various endometrial receptivity markers [19].

\section{Coagulation alterations}

Among the different clinical manifestations that CD can present with, we found thromboembolic phenomena which represent a puzzle with multiple and varied pieces such as hyperhomocysteinemia, B12 and/or folate deficiency, methylenetetrahydrofolate reductase mutations, and protein $C$ and $S$ deficiency resulting from vitamin $K$ deficiency [20]. Recent studies linked adverse pregnancy outcomes to acquired or hereditary thrombophilia [21,22]. Specifically, a higher incidence of early recurrent abortion is associated with the methyleneterahydrofolate reductase (MTHFR) C677T and with the prothrombin G202010A variants [23], and with factor V Leiden [24].

Moreover, a 4G variant of plasminogen activator inhibitor-1 gene was shown to be likely capable of affecting placental and/or fetal microvascular function in a way that may lead to miscarriage [25]. 


\section{Immune-mediated mechanisms}

Although all of these findings can help to shed light on the intricate pathogenic mechanisms underlying the alterations in fertility and pregnancy seen in $C D$, nowadays the most promising field of research on the matter appears to be that of autoimmunity. CD is an autoimmune disease in which the ingestion of gluten triggers the production of circulating antitransglutaminase (anti-tTG) autoantibodies.

A study by Di Simone, et al. [26] showed that circulating anti-tTG antibodies are responsible for impairing the placental function. It this study human primary trophoblastic cells, isolated from term placenta, were exposed to anti-tTG immunoglobulin $\mathrm{G}$ ( $\operatorname{lgG}$ ) antibodies in vitro. Trophoblast has a pivotal role in ensuring the synchronized interaction in the adhesion and invasive events that occurs at the embryomaternal interface after implantation and its differentiation is characterized by the development of extravillous trophoblast (EVT) that migrates into the maternal myometrium. Anti-tTG IgG showed a specific dose- and time-dependent binding to human trophoblast, which after being exposed to such autoantibodies showed an impaired invasiveness, a decreased activity of cellular matrix metalloprotease (MMP) and greater percentage of cellular apoptosis assessed by the expression of indicators of trophoblast damage such as TdT-mediated dUTP digoxigenin nick end labeling (TUNEL) and annexine V.

Deeper understanding of the subject has been reached in a study by Di Simone, et al. [27] that focused on the effect of circulating anti-tranglutaminase type 2 (anti-TG2) autoantibodies on the process of endometrial angiogenesis and decidualization which are prerequisites for placental development. In particular the study showed how anti-TG2 antibodies bind to human endometrial endothelial cells (HEECs) and decrease newly formed vessels both in vitro and in vivo. Impaired angiogenesis is due to the ability of anti-TG2 antibodies to inhibit the activation of HEEC matrix metalloprotease-2 (MMP-2), to disarrange cytoskeleton fibers, to change the physical and mechanical properties of cell membranes and to inhibit the intracellular phosphorylation of FAK and ERK. Anti-TG2 were thus found to inhibit endometrial angiogenesis by altering TG2-dependent migration of HHECS and extracellular matrix degeneration.

Furthermore, a study by Sóñora, et al. [28] investigated the effects of anti-tTG autoantibodies from sera of patients with CD on cytotrophoblast cell line (Swan-71 cells). Such cells were taken as a model of the syncytial microvillous surface of human placenta where tTG localizes. At this site tTG contributes to injury healing during the early implantation period characterized by important tissue remodeling and changes. The study demonstrated that anti-tTG antibodies reduce proliferation rate and migration of trophoblast cells, promote apoptosis levels and are able to interfere with the clearance of trophoblast apoptotic bodies through a mechanism involving MFG-E8 (milk fat globuline - EGF factor 8)-tTG interaction.

\section{Endometrium inflammation}

A study by D'Ippolito, et al. [29] showed for the first time a higher proportion of HLA DQ2/DQ8 positivity in women with Recurrent Pregnancy Loss (RPL) as compared to controls (fertile women). HLA DQ2/DQ8 haplotypes codify for the $\mathrm{DQ} 2 / \mathrm{DQ} 8$ proteins that, in celiac patients, are responsible for presenting the immunogenic gluten peptides to DQ2/ DQ8-restricted CD4+ T cells. Once activated, CD4+ T cells trigger a complex immune response that leads to increased production of interferon (INF) $\gamma$, tumor necrosis factor (TNF) $\alpha$, and autoantibodies like anti-tTG, anti-endomysium and anti-gliadin antibodies. This process will eventually lead to the disruption of tight junction in the intestinal epithelium generating a leaky gut condition.

Interestingly, a study by Tersigni, et al. [30] showed an existing linkage between endometrial NALP-3 inflammasome over expression and activation, and a leaky gut condition in woman with idiopathic RPL. Thus, it has been hypothesized that leaky gut, occurring for reasons not fully understood, allows the passage of antigens through the intestinal barrier that might lead to inflammation of the endometrium. Further studies are needed to determine whether or not there is a connection among HLA DQ2/DQ8, leaky gut and endometrial inflammation in women with adverse pregnancy outcomes and $C D$.

\section{Conclusion}

Although numerous studies have demonstrated an association between $\mathrm{CD}$ and gynecological/obstetric disorders and the potential pathogenic mechanisms underlying such linkage. Although it has been widely demonstrated by numerous studies the association between $C D$ and gynecological/obstetric disorders such as infertility, menstrual cycle disorders and adverse pregnancy outcomes, the understanding of the pathogenic mechanisms of this conditions is still in its infancy. Multiple factors such as malnutrition due to intestinal malabsorption, coagulation alterations, endometrial inflammation and immune-mediated mechanisms have been shown to lead to the complex and different alterations seen in patients with $C D$ and adverse pregnancy outcomes. Current knowledge on how such mechanisms act and interact is insufficient and thus further studies are needed. Ultimately, it is hoped that a thorough understanding of CD will yield new, rationally designed therapeutic approaches that will improve pregnancy outcomes for CD women through personalized medicine.

\section{References}

1. Saccone G, Berghella V, Sarno L, et al. (2016) Celiac disease and obstetric complications: A systematic review and metaanalysis. Am J Obstet Gynecol 214: 225-234.

2. Tersigni C, Castellani R, de Waure C, et al. (2014) Celiac disease and reproductive disorders: Meta-analysis of epidemiologic associations and potential pathogenic mechanisms. Hum Reprod Update 20: 582-593.

3. Eliakim R, Sherer DM (2001) Celiac disease: Fertility and pregnancy. Gynecol Obstet Invest 51: 3-7.

4. Butler MM, Kenny LC, McCarthy FP (2011) Coeliac disease and pregnancy outcomes. Obstet Med 4: 95-98.

5. Morris JS, Adjukiewicz AB, Read AE (1970) Coeliac infertility: An indication for dietary gluten restriction? Lancet 1: 213-214. 
6. Casella G, Orfanotti G, Giacomantonio L, et al. (2016) Celiac disease and obstetrical-gynecological contribution. Gastroenterol Hepatol Bed Bench 9: 241-249.

7. Collins P, Vilska S, Heinonen PK, et al. (1996) Infertility and celiac disease. Gut 39: 382-384.

8. Sher KS, Mayberry JF (1994) Female fertility, obstetric and gynaecological history in coeliac disease. A case control study. Digestion 55: 243-246.

9. Ferguson R, Holmes GK, Cooke WT (1982) Coeliac disease, fertility and pregnancy. Scand J Gastroenterol 17: 65-68.

10. Gasbarrini A, Torres ES, Trivellini C, et al. (2000) Recurrent spontaneous abortion and intrauterine fetal growth retardation as symptoms of coeliac disease. Lancet 356: 399-400.

11. Ciacci C, Cirillo M, Auriemma G, et al. (1996) Celiac disease and pregnancy outcome. Am J Gastroenterol 91: 718-722.

12. Salvatore S, Finazzi S, Radaelli G, et al. (2007) Prevalence of undiagnosed celiac disease in the parents of preterm and/or small for gestational age infants. Am J Gastroenterol 102: 168-173.

13. Ludvigsson JF, Montgomery SM, Ekbom A (2005) Celiac disease and risk of adverse fetal outcome: A population-based cohort study. Gastroenterology 129: 454-463.

14. Moleski SM, Lindenmeyer CC, Veloski JJ, et al. (2015) Increased rates of pregnancy complications in women with celiac disease. Ann Gastroenterol 28: 236-240.

15. Peracchi M, Molteni N, Cantalamessa L, et al. (1992) Abnormal growth hormone responsiveness to stimuli in women with active celiac sprue. Am J Gastroenterol 87: 580-583.

16. Bedwal RS, Bahuguna A (1994) Zinc, Copper and selenium in reproduction. Experienntia 50: 626-640.

17. Wei SQ, Fraser W, Luo ZC (2010) Inflammatory cytokines and spontaneous preterm birth in asymptomatic women: A systemic review. Obstet Gynecol 116: 393-401.

18. O’Keeffe J, Mills K, Jackson J, et al. (1999) T cell proliferation, MHC class II restriction and cytokine products of gliadinstimulated peripheral blood mononuclear cells (PBMC). Clin Exp Immunol 117: 269-276.

19. Banerjee P, Jana SK, Pasricha P, et al. (2013) Proinflammatory cytokines induced altered expression of cyclooxygenase- 2 gene results in unreceptive endometrium in women with idiopathic recurrent spontaneous miscarriage. Fertil Steril 99: 179-187.

20. Aaron Lerner, Nancy Agmon-Levin, Yinon Shapira, et al. (2013) The thrombophilic network of autoantibodies in celiac disease. BMC Med 11: 89.

21. Vossen CY, Preston FE, Conard J, et al. (2004) Hereditary thrombophilia and fetal loss: A prospective follow-up study. J Thromb Haemost 2: 592-596.

22. Kovalevsky G, Gracia CR, Berlin JA, et al. (2004) Evaluation of the association between hereditary thrombophilias and recurrent pregnancy loss: A meta-analysis. Arch Intern Med 164: 558-563.

23. Martinelli I, Taioli E, Cetin I, et al. (2000) Mutations in coagulation factors in women with unexplained late fetal loss. New Engl J Med 343: 1015-1018.

24. Ridker PM, Miletich JP, Buring JE, et al. (1998) Factor V Leiden mutation as a risk factor for recurrent pregnancy loss. Ann Intern Med 128: 1000-1003.

25. Ciacci C, Tortora R, Scudiero O, et al. (2009) Early pregnancy loss in celiac women: The role of genetic and markers of thrombophilia. Dig Liver Dis 41: 717-720.

26. Di Simone N, Silano M, Castellani R, et al. (2010) Anti-tissue transglutaminase antibodies from celiac patients are responsible for trophoblast damage via apoptosis in vitro. Am J Gastroenterol 105: 2254-2261.

27. Di Simone N, De Spirito M, Di Nicuolo F, et al. (2013) Potential new mechanisms of placental damage in celiac disease: antitransglutaminase antibodies impair human endometrial angiosenesis. Biol Reprod 89: 88.

28. Sóñora C, Calo G, Fraccaroli L, et al. (2014) Tissue transglutaminase on trophoblast cells as a possible target of autoantibodies contributing to pregnancy complications in celiac patients. Am J Reprod Immunol 72: 485-495.

29. D'Ippolito S, Gasbarrini A, Caastellani R, et al. (2016) Human leukocyte antigen (HLA) DQ2/DQ8 prevalence in recurrent pregnancy loss women. Autoimmun Rev 15: 638-643.

30. Tersigni C, D'Ippolito S, Di Nicuolo F, et al. (2018) Recurrent pregnancy loss is associated to leaky gut: A novel pathogenic model of endometrium inflammation? J Transl Med 16: 102.

DOI: $10.36959 / 621 / 597$

Copyright: (c) 2018 Simone ND, et al. This is an open-access article distributed under the terms of the Creative Commons Attribution License, which permits unrestricted use, distribution, and reproduction in any medium, provided the original author and source are credited. 